\title{
Prevalence and Associated Factors of Depression among Admitted Adult Patients in Surgical and Medical Wards of Saint Paul's Hospital Millennium Medical College, Addis Ababa, Ethiopia
}

\author{
Merga Siyoum, ${ }^{1}$ Getachew Assfaw, ${ }^{1}$ Henok Yitbark, ${ }^{1}$ and Getachew Tesfaw $\mathbb{D}^{2}$ \\ ${ }^{1}$ Research and Training Department, Amanuel Mental Specialized Hospital, Addis Ababa, Ethiopia \\ ${ }^{2}$ Department of Psychiatry, College of Medicine and Health Science, University of Gondar, Gondar, Ethiopia \\ Correspondence should be addressed to Getachew Tesfaw; getachewtesfaw@gmail.com
}

Received 31 July 2020; Accepted 6 January 2021; Published 31 January 2021

Academic Editor: Janusz K. Rybakowski

Copyright ( 2021 Merga Siyoum et al. This is an open access article distributed under the Creative Commons Attribution License, which permits unrestricted use, distribution, and reproduction in any medium, provided the original work is properly cited.

\begin{abstract}
Background. Depression is a leading cause of major public health problems globally, and its prevalence has been increasing, particularly in low- and middle-income countries including our country. Therefore, this study is aimed at exploring depression symptoms and their determinants among admitted medical and surgical patients which is important to get optimal care for patients. Methods. An institution-based cross-sectional study was conducted from May to June 2019, on adults' medical and surgical admitted patients at Saint Paul's Hospital Millennium Medical College, Addis Ababa, Ethiopia. The systematic random sampling technique was used to get a total of 590 samples. The standardized hospital anxiety and depression scale (HADS) was used to assess individual depression symptoms. We computed the bivariate and multivariate binary logistic regression analyses to identify factors associated with depression symptoms. Statistical significance was declared at $P<0.05$. Result. The prevalence of depression symptoms was found to be $53.9 \%$ (95\% CI: 50.2, 57.0). In the multivariable logistic regression, female sex $(\mathrm{AOR}=2.04,95 \% \mathrm{CI}: 1.35,3.09)$, being single $(\mathrm{AOR}=3.65,95 \% \mathrm{CI}: 3.48,2.10,5.78)$, widowed $(\mathrm{AOR}=2.82,95 \% \mathrm{CI}: 1.27$, $6.30)$, unable to read and write $(\mathrm{AOR}=2.71,95 \% \mathrm{CI}: 1.14,6.47)$, admission at medical ward $(\mathrm{AOR}=1.59,95 \% \mathrm{CI}: 1.02,2.46)$, history of mental illness $(\mathrm{AOR}=1.59,95 \% \mathrm{CI}: 1.02,2.46)$, and poor social support ( $\mathrm{AOR}=2.82,95 \% \mathrm{CI}$ : 1.57, 5.11) were factors significantly associated with depression symptoms. Conclusion. The prevalence of depression symptoms among admitted patients was high. Female sex, being single, widowed, unable to read and write, admission at medical ward, history of mental illness, and poor social support were factors significantly associated with depression symptoms. It is better for the Ministry of Health to give training on how to screen depression among medical and surgical patients, and interventions that would be addressing the awareness of the above factors would be beneficial to prevent further complications.
\end{abstract}

\section{Introduction}

Depression is a common mental illness globally characterized by sadness and loss of interest in activities that are normally enjoyable, accompanied by an inability to carry out daily functions $[1,2]$. According to the World Health Organization report, more than three hundred twenty-two million people are living with depression in the world [3]. And it is a leading cause of disability and ill health globally [2-5]. It is the third leading cause of disease burden, and it accounts for $4.3 \%$ of total disability [6]. Lifetime and one-year prevalence of depression are approximately $13 \%$ and $5-7 \%$, respectively $[5,7]$.

In 2017, the WHO report showed that the prevalence of depression ranged from 9 to $27 \%$ across different regions $[3,8]$. In sub-Sahara, the magnitude of depression ranges from 15 to $30 \%$, and in Ethiopia, it accounts for 9.1\% $[9,10]$. It is more common in females than males $[1,2,9]$. It contributes to its huge public health burden, including the impact of depression which increased the risk of suicide and premature mortality from cooccurring physical disorders $[5,11]$. 
The WHO 2014 report revealed that the rate of depression among patients with physical illness was high, and it was two to three times more common in people with physical problems $[3,12]$. Depression has a high prevalence among adult patients in clinical settings, which ranges from 5 to $30 \%[13,14]$. But it was high among admitted chronic medical patients [15]. Two meta-analyses were done across different countries among adult admitted individuals; the prevalence of depression was 15 to $60 \%$ and $9.6 \%$ to $16.5 \%$, respectively $[16,17]$.

Several factors could play a role in the development of depression secondary to medical and surgical illnesses. These include repeated hospital admission history, poor social support, long hospital stay, previous history of mental illness, female sex, and admission in surgical or medical ward [18-22]. The comorbidity of depression with admitted medical and surgical patients negatively impacts the quality of life, functioning, adherence to medication, and high risk of suicide and increased morbidity and mortality [23, 24].

The vast majority of people with depression symptoms do not receive any care in many countries. Even in developed countries, nearly half of people with depression do not get treatment $[2,4]$. The prevalence of depression is increasing, particularly in low- and middle-income countries including our country $[6,13]$.

However, research into depression symptoms and their determinants among people with medical and surgical patients in low- and middle-income countries is limited. Therefore, the aim of this study was to identify the prevalence of depression symptoms and associated factors among people with medical and surgical patients in Ethiopia which has a pivotal role in further intervention.

\section{Methods and Materials}

2.1. Study Setting and Period. An institution-based crosssectional study was conducted from May to June 2019 at St. Paul's Hospital Millennium Medical College, Addis Ababa, Ethiopia. The medical school opened in 2007, but the hospital was established in 1968 by the late Emperor Haile Selassie. The hospital has both inpatient and outpatient services with 400 beds, and 2682 patients are admitted to the wards each month, especially 884 patients from the medical ward and 379 from the surgical ward. So, a total of 1263 participants were admitted to the medical and surgical wards per month.

2.2. Study Population. All admitted adult patients aged $\geq 18$ were included, while patients found to be unconscious and unable to take consent were excluded.

2.3. Sample Size Calculation and Sampling Procedure. The sample size was calculated by using the single population proportion formula with $95 \%$ confidence interval (CI) and a $4 \%$ margin of error and taking the prevalence of depression $38 \%$ from a previously published study in our country [25]. This yields a total of 566 samples. Having assumed a 10\% nonrespondent rate, the final sample size was $566+57=$ 623 admitted medical and surgical patients recruited randomly by using a systematic sampling technique. The sam- pling interval was determined by dividing the total study participants who were admitted in medical and surgical wards during the month of data collection by the total sample size. Proportionally, 436 and 187 admitted patients were taken from medical and surgical wards, respectively; so, the selection skip interval for both wards was two. The first individual was selected by a lottery method from the admission register.

2.4. Data Collection. Data were collected using a pretested interviewer-administered questionnaire which contained depression as the dependent variable and several other determinant variables, including sociodemographic factors, clinical factors, social support, and substance use.

2.4.1. Instruments. Social support was assessed by using the Oslo 3-item social support scale and used several studies. It provides a brief measure of social support and functioning and is considered to be one of the best predictors of mental health. It covered different levels of social support by measuring the number of people the respondents feel close to, the interest and concerns showed by others, and the ease of obtaining practical help from others. The sum score scale ranged from 3 to 14 and had three broad categories: "poor social support" 3-8, "moderate support" 9-11, and "strong support" 12-14, respectively [26]. The internal consistency of the Oslo social support scale in this study was 0.83 . It has been used in Ethiopia in different clinical settings $[22,27,28]$. Substance use was assessed by using the WHO Alcohol, Smoking, and Substance Involvement Screening Test (ASSIST) guidelines for use in primary care. It is a brief screening questionnaire to find out people's use of psychoactive substance. It provides information about the substances people have ever used in their lifetime and at least one of any specific substances for nonmedical purpose in the past three months, respectively. Its Cronbach' alpha is $80 \%$, sensitivity $0.80 \%$, and specificity $71 \%$, respectively [29]. Income was assessed by using the WHO poverty line which is less than or equal to 1.9 dollars per day as extreme poverty and greater than 1.9 dollars per day as the poverty line which is 1627 according to Ethiopian Birr during the study period [30].

Depression was assessed by using HADS (hospital anxiety and depression scale) which has 7-item questionnaire subscales for depression symptoms. Possible scores range from 0 to 21 for depression with cutoff points of greater than or equal to eight. In Ethiopia, it was validated, and its internal consistency was 0.76 for depression subscales [31].

2.5. Data Processing and Analysis. Data were entered into Epi-data software version 3.1 and imported to SPSS version 20 for analysis. Univariate, bivariate, and multivariate logistic regression analyses were done to see the association of each independent variable with the dependent variable. The strength of associations was evaluated using the adjusted odds ratio with a $95 \% \mathrm{CI}$, and a $P$ value of less than 0.05 was considered statistically significant.

2.6. Ethical Considerations. Ethical approval was obtained from the Institutional Review Board (IRB) of the University 
TABLE 1: Distributions of sociodemographics of participants admitted in medical and surgical wards at SPHMMC, $2019(n=590)$.

\begin{tabular}{|c|c|c|c|}
\hline Variables & Category & Frequency & Percent \\
\hline Age & Mean and SD & $39.71( \pm 14.94)$ & \\
\hline \multirow{2}{*}{ Sex } & Male & 301 & 51 \\
\hline & Female & 289 & 49 \\
\hline \multirow{2}{*}{ Residence } & Urban & 345 & 58.5 \\
\hline & Rural & 245 & 41.5 \\
\hline \multirow{4}{*}{ Marital status } & Married & 276 & 46.8 \\
\hline & Single & 231 & 39.2 \\
\hline & Divorced & 39 & 6.6 \\
\hline & Widowed/separated & 44 & 7.5 \\
\hline \multirow{4}{*}{ Ethnicity } & Amhara & 248 & 42.0 \\
\hline & Oromo & 254 & 43.1 \\
\hline & Gurage & 58 & 9.8 \\
\hline & Others* & 30 & 5.1 \\
\hline \multirow{4}{*}{ Religion } & Orthodox & 329 & 55.8 \\
\hline & Protestant & 126 & 21.4 \\
\hline & Muslim & 105 & 17.8 \\
\hline & Catholic and others & 30 & 5.1 \\
\hline \multirow{5}{*}{ Educational level } & Unable to read and write & 219 & 37.1 \\
\hline & Can read and write only & 92 & 15.6 \\
\hline & Primary school & 152 & 25.8 \\
\hline & Secondary school & 84 & 14.2 \\
\hline & Above diploma & 43 & 7.3 \\
\hline \multirow{7}{*}{ Jobs } & Farmer & 145 & 24.6 \\
\hline & Housewife & 114 & 19.3 \\
\hline & Merchant & 35 & 5.9 \\
\hline & Governmental employer & 62 & 10.5 \\
\hline & Private & 111 & 18.8 \\
\hline & Student & 36 & 6.1 \\
\hline & Daily worker & 87 & 14.7 \\
\hline \multirow{5}{*}{ Living condition } & With husband or wife & 276 & 46.8 \\
\hline & With family & 100 & 16 \\
\hline & With relatives & 35 & 5.9 \\
\hline & Alone & 145 & 24.6 \\
\hline & With others** & 34 & 5.8 \\
\hline \multirow{2}{*}{ Monthly income } & $\leq 1627$ & 377 & 63.9 \\
\hline & $>1627$ & 213 & 36.1 \\
\hline
\end{tabular}

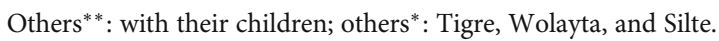

of Gondar. A letter of permission was issued by Amanuel Mental Specialized Hospital. We received informed written consent from study participants. Confidentiality was maintained by omitting personal identifiers.

\section{Results}

3.1. Sociodemographic Characteristics. Out of a total of 623 participants, 590 completed the survey with a response rate of $94.7 \%$. The mean age of the participants was 39.71 $( \pm 14.94)$ years, and above half $(51 \%)$ of the participants were male. Nearly three-fifths (58.5\%) were from urban residents. Out of the participants, 329 (55.8\%) were Orthodox Christian followers, and $276(46.8 \%)$ were married. Almost half of the study participants $(46.8 \%)$ were living with their husband or wife, 219 (37.1\%) were unable to read and write, 145 (24.6\%) were farmers, and more than three-fifths (63.9\%) have gotten $\leq 1627$ Ethiopian Birr per monthly income (Table 1). 
TABLE 2: Distribution of clinical factors of respondents admitted at medical and surgical wards at SPHMMC, Addis Ababa, Ethiopia, $2019(n=590)$.

\begin{tabular}{|c|c|c|c|}
\hline Variables & Categories & Frequency & Percent \\
\hline \multirow{2}{*}{ Site admission } & Medical ward & 410 & 69.5 \\
\hline & Surgical ward & 180 & 30.5 \\
\hline \multirow{6}{*}{ Current diagnosis } & Musculoskeletal d/r & 41 & 6.9 \\
\hline & Genitourinary d/r & 56 & 9.5 \\
\hline & Endocrine d/r & 185 & 31.4 \\
\hline & Gastrointestinal d/r & 103 & 17.5 \\
\hline & Infectious diseases & 155 & 26.3 \\
\hline & Cardiovascular $\mathrm{d} / \mathrm{r}$ & 50 & 8.5 \\
\hline \multirow{4}{*}{ Duration of hospital stay } & One week & 251 & 42.5 \\
\hline & Two weeks & 242 & 41.0 \\
\hline & Three weeks & 41 & 6.9 \\
\hline & $\geq$ one month & 56 & 9.5 \\
\hline \multirow{2}{*}{ Pervious history of admission } & No & 372 & 63.1 \\
\hline & Yes & 218 & 36.9 \\
\hline \multirow{2}{*}{ Multiple diagnoses of medical illness } & No & 41 & 70.3 \\
\hline & Yes & 17 & 29.7 \\
\hline \multirow{2}{*}{ History of suicidal attempt } & No & 527 & 89.3 \\
\hline & Yes & 63 & 10.7 \\
\hline \multirow{2}{*}{ Feeling of pain } & No & 285 & 48.3 \\
\hline & Yes & 305 & 51.7 \\
\hline \multirow{2}{*}{ Fear of death } & No & 488 & 82.9 \\
\hline & Yes & 102 & 17.3 \\
\hline \multirow{2}{*}{ Fear of complication } & No & 248 & 42.0 \\
\hline & Yes & 342 & 58 \\
\hline \multirow{2}{*}{ History of mental illness } & No & 46 & 78.8 \\
\hline & Yes & 125 & 21.2 \\
\hline \multirow{2}{*}{ Family history of mental illness } & No & 480 & 81.4 \\
\hline & Yes & 11 & 18.6 \\
\hline \multirow{2}{*}{ Family history of chronic medical illness } & Yes & 89 & 15.1 \\
\hline & No & 501 & 84.9 \\
\hline
\end{tabular}

3.2. Clinical Characteristics of Respondents. Of the 590 participants, $410(69.5 \%)$ were admitted to medical wards, onethird $31.4 \%$ was diagnosed with endocrine disorder, and two-fifths $(42.5 \%)$ of them were staying in the hospital for a week. Almost two-fifths (63.1\%) of the participants had a previous history of admission, 415 (70.3\%) of the participants had multiple medical diagnoses, and about $63(10.7 \%)$ of the respondents had a history of suicidal attempts (Table 2).

3.3. Social and Substance-Related Factors. Among the total of the participants, two-fifths 239 (40.5\%) had poor social support. Three-fourths $(76.8 \%)$ of the respondents had a history of lifetime substance use, 404 (68.5\%) have been drinking alcohol, $88(14.9 \%)$ were smoking cigarettes, and nearly $33.9 \%$ were chewing khat throughout their lifetime, respec- tively. At the moment, $26.1 \%$ of respondents have a history of current substance use (Table 3).

3.4. Prevalence of Depression among Respondents. The prevalence of depression symptoms among study participants was $53.9 \%$ with a $95 \%$ CI $(50.20,57.00)$. Over three-fifths (61.9\%) were females, and more than two-fifths (46.2\%) were male.

3.4.1. Factors Associated with Depression Symptoms. Of the independent variables, female sex, being single, being widowed/r, unable to read and write, daily labor, living alone, admitted to medical ward, history of previous admission, history of mental illness, and poor social support yielded $P$ value less than 0.2 in the bivariate logistic regression and were considered in the multivariate logistic regression model. 
TABle 3: Psychosocial and substance use characteristics of respondents admitted at medical and surgical wards of SPHMMC, Addis Ababa, Ethiopia, $2019(n=590)$.

\begin{tabular}{|c|c|c|c|}
\hline Variables & Categories & Frequency & Percent \\
\hline \multirow{3}{*}{ Social support } & Poor & 239 & 40.5 \\
\hline & Moderate & 266 & 45 \\
\hline & Good & 85 & 14 \\
\hline \multirow{2}{*}{ Ever substance use } & Yes & 453 & 76.8 \\
\hline & No & 137 & 23.2 \\
\hline \multirow{2}{*}{ Ever khat use } & Yes & 200 & 33.9 \\
\hline & No & 390 & 66.1 \\
\hline \multirow{2}{*}{ Ever alcohol use } & Yes & 404 & 68.5 \\
\hline & No & 186 & 31.5 \\
\hline \multirow{2}{*}{ Ever cigarette use } & Yes & 88 & 14.9 \\
\hline & No & 502 & 85.1 \\
\hline \multirow{2}{*}{ Ever other substance use } & Yes & 22 & 3.7 \\
\hline & No & 568 & 96.3 \\
\hline \multirow{2}{*}{ Current addictive substance use } & Yes & 175 & 29.7 \\
\hline & No & 415 & 70.3 \\
\hline \multirow{2}{*}{ Current khat use } & Yes & 46 & 7.8 \\
\hline & No & 544 & 92.2 \\
\hline \multirow{2}{*}{ Current alcohol use } & Yes & 154 & 26.1 \\
\hline & No & 436 & 73.9 \\
\hline \multirow{2}{*}{ Current other addictive substance use } & Yes & 10 & 1.7 \\
\hline & No & 580 & 98.3 \\
\hline
\end{tabular}

The multivariate analysis suggested that female sex are two times more likely to develop depression symptoms compared to male sex $(\mathrm{AOR}=2.04,95 \% \mathrm{CI}: 1.35,3.09)$. Being single was over three times to develop depression symptoms compared to their counterparts $(\mathrm{AOR}=3.65,95 \% \mathrm{CI}$ : 3.48 , $2.10,5.78$ ), and widower/separated was 2.8 times more likely to be risky to depression when compared to married $(\mathrm{AOR}=2.82,95 \% \mathrm{CI}: 1.27,6.30)$. The odds of developing depression symptoms were 2.7 times higher among those unable to read and write when compared with college or university graduates $(\mathrm{AOR}=2.71,95 \% \mathrm{CI}: 1.14,6.47)$. The odds of developing depression were more than 1.5 times more likely among patients admitted in medical wards as compared with those who are admitted in surgical wards ( $\mathrm{AOR}=1.59,95 \% \mathrm{CI}: 1.02,2.46)$. History of mental illness has 1.79 times increased the risk of depression compared to patients who had no history of mental illness $(\mathrm{AOR}=1.79$, 95\% CI: 1.06, 3.05), and respondents who had poor social support were 2.82 times more likely to lead to depression as compared to respondents who had strong social support $(\mathrm{AOR}=2.82,95 \% \mathrm{CI}: 1.57,5.11)$ (Table 4).

\section{Discussion}

In the current study, the prevalence of depression symptoms and their possible association with various factors were assessed. The results revealed that a high proportion of depression symptoms were found among people admitted in medical and surgical wards. The prevalence of depression symptoms among people admitted in medical and surgical wards was found to be $53.9 \%$.

Regarding prevalence, our result is in line with those of other two studies carried out in Northwest and Eastern Ethiopia which reported that the prevalence of depression symptoms was $54.6 \%$ and $57.9 \%$, respectively $[32,33$. However, this finding is lower than those of studies conducted in India $60.5 \%$ [17] and Iran 58.8\% [19]. The possible reason for the variation of the above prevalence might be due to the discrepancy of instruments, types of diagnosis and site of admission, study population contrasts, and the sociocultural difference between Ethiopia and the other countries.

On the other hand, our finding is higher than those of studies done in Ethiopia 38\% [25], Nigeria 45.3\% [34], Iran $42.3 \%$ [21], Italy 21\% [17], England 38\% [35], Jamaica $33.3 \%$ [36], and Brazil 28\% [37]. The variation in the above rates might be due to differences in the sample sizes, age of participants, types of study design, the use of various scales and rating for assessing the level of depression symptoms, methodologies, types of admission site, types of patients, and sociocultural contrasts between Ethiopia and other countries.

There were different factors associated with depression symptoms in this study; female gender is two times more likely risky for depression symptoms when compared to male 
TABLE 4: Bivariate and multivariate analyses among admitted patients in medical and surgical wards at SPHMMC, Addis Ababa, Ethiopia $(n=590)$.

\begin{tabular}{|c|c|c|c|c|}
\hline \multirow{2}{*}{ Variable } & \multicolumn{2}{|c|}{ Depression } & \multirow{2}{*}{ COR $(95 \%$ CI $)$} & \multirow{2}{*}{ AOR (95\%) } \\
\hline & Yes & No & & \\
\hline \multicolumn{5}{|l|}{ Sex } \\
\hline Male & 139 & 162 & 1 & 1 \\
\hline Female & 179 & 110 & $1.89(1.37,2.63)$ & $2.04(1.35,3.08)^{*}$ \\
\hline \multicolumn{5}{|l|}{ Marital status } \\
\hline Married & 107 & 169 & 1 & 1 \\
\hline Single & 159 & 72 & $3.49(2.41,5.04)$ & $3.48(2.09,5.77)^{* *}$ \\
\hline Divorced & 25 & 14 & $2.82(1.40,5.67)$ & $2.28(0.96,5.42)$ \\
\hline Widowed/separated & 27 & 17 & $2.51(1.31,4.82)$ & $2.82(1.26,6.29)^{*}$ \\
\hline \multicolumn{5}{|l|}{ Educational level } \\
\hline Unable to read and write & 149 & 70 & $2.97(1.51,5.77)$ & $2.71(1.14,6.45)^{*}$ \\
\hline Can read and write only & 51 & 41 & $1.73(0.83,3.59)$ & $2.28(0.90,5.79)$ \\
\hline Primary school & 62 & 90 & $0.97(0.48,1.90)$ & $1.06(0.44,2.56)$ \\
\hline Secondary & 38 & 46 & $1.15(0.55,2.41)$ & $1.27(0.50,3.23)$ \\
\hline College/university & 18 & 25 & 1 & 1 \\
\hline \multicolumn{5}{|l|}{ Living conditions } \\
\hline Wife/husband & 127 & 149 & 1 & 1 \\
\hline Family & 46 & 54 & $0.99(0.63,1.58)$ & $0.78(0.45,1.35)$ \\
\hline Relatives & 20 & 15 & $1.56(0.77,3.18)$ & $0.71(0.29,1.68)$ \\
\hline Alone & 106 & 39 & $3.19(2.06,4.94)$ & $1.46(0.80,2.67)$ \\
\hline Others* & 19 & 15 & $1.49(0.73,3.04)$ & $0.65(0.28,1.54)$ \\
\hline \multicolumn{5}{|l|}{ Site admission } \\
\hline Medical ward & 233 & 177 & $1.47(1.04,2.09)$ & $1.59(1.02,2.47)^{*}$ \\
\hline Surgical ward & 85 & 95 & 1 & 1 \\
\hline \multicolumn{5}{|l|}{ Previous history of admission } \\
\hline Yes & 144 & 74 & $2.21(1.57,3.13)$ & $1.42(0.94,2.16)$ \\
\hline No & 174 & 198 & 1 & 1 \\
\hline \multicolumn{5}{|l|}{ History of mental illness } \\
\hline Yes & 92 & 33 & $2.95(1.90,4.57)$ & $1.79(1.06,3.05)^{*}$ \\
\hline No & 226 & 239 & 1 & 1 \\
\hline \multicolumn{5}{|l|}{ Social support } \\
\hline Poor & 166 & 73 & $2.68(1.61,4.46)$ & $2.82(1.56,5.11)^{*}$ \\
\hline Moderate & 113 & 153 & $0.87(0.53,1.42)$ & $0.86(0.49,1.51)$ \\
\hline Good & 39 & 46 & 1 & 1 \\
\hline
\end{tabular}

Note: ${ }^{* *}<0.001{ }^{*}<0.05$. Others ${ }^{* *}$ : with their children; Hosmer and Lemeshow Test $=0.52$.

sex among patients admitted to medical and surgical wards. In this finding, $30 \%$ of females had depression which was higher than $23.6 \%$ of males. This is supported by studies done in Brazil among admitted medical patients; female sex had more than one and a half times of depression symptoms than male sex [38], and in Iran, female gender is significantly associated with depression symptoms among surgical patients compared with male sex [19]. Females are nearly twice as likely as men to be vulnerable to depression symptoms. Some mood changes and depressed feelings occur with normal hormonal changes. There are different factors that may increase the risk of depression symptoms in females such as biological factors, personal circumstances, and different countries with various sociocultural backgrounds. For example, in Ethiopia, female sex experiences some form of trauma during their lives like gender violence, discrimination, and mistreatment of women associated with medical or surgical illness leading to high stress, social withdrawal, and low self-esteem which in turn increased depression symptoms. 
Being single was over three times likely to have depression symptoms compared to their counterpart, and widowed was 2.8 times more likely risky to depression when compared to married. This is consistent with other studies carried out in Uganda [39], China [40], and England [35]. Being widowed was associated with psychological distress among adults admitted to medical and surgical wards [39], living alone without their family members was highly associated among admitted patients [39], and being widowed has increased depression symptoms among admitted patients [40].

This study revealed that unable to read and write has nearly three times risk of depression symptoms compared to their counterparts, which is in line with other studies done in Iran; a lower educational level was significantly associated with depression symptoms among surgical patients [19], and in Brazil, a lower level of education has more than three times risk of depression among individuals admitted in a general hospital [41].

Adult patients admitted in medical wards are more than 1.5 times more likely to have depression as compared with those who are admitted in the surgical and medical wards. Depression symptoms are a common condition among medical patients, and its symptoms lead to increased morbidity and mortality rates, psychosocial functioning, and increased use of medical resources. But different studies revealed that depression symptoms are highly associated with admission in the surgical ward than the medical ward $[32,33,42]$. So, it needs further research, why patients admitted in medical wards are highly risky for depression symptoms compared to surgical ward patients.

This study showed that depression symptoms were significantly associated with prior history of mental illness. History of mental illness has 1.79 times increased the risk of depression symptoms compared to patients who had no history of mental illness. This was supported by a study done in Iran; a previous history of mental illness was a risk factor for depression symptoms in surgical patients [19].

Poor social support was nearly three times more likely to have depression symptoms compared to those who have good social support in this study that was comparable with the studies done in Ethiopia; poor social support has two times more likely risk to develop depression compared with good social support [27]; in another study in Ethiopia, poor social support has more than three times increased depression symptoms among medical patients [22], and in Wuhan, China, the absence of medical insurance and poor family support were highly associated with depression symptoms among admitted patients [40], and in Iran, lack of family support was a risk factor for depression disorders [19]. The present study has several strengths. Firstly, we used standardized instruments to measure depression symptoms; secondly, we adjusted the final multivariate model for the most important factors affecting depression. This study also has limitations; it was a cross-sectional study and thus cannot establish a causal link between associated factors and depression symptoms among admitted medical and surgical patients. The finding is likely only to hint at the complex interactions between depression symptoms and explanatory variables. So, the interpretation and usage of the study must be considered a limitation.

\section{Conclusion}

The prevalence of depression symptoms among admitted patients in medical and surgical wards was found to be $53.9 \%$. Depression was significantly associated with female sex, being single, widowed, unable to read and write, site of admission, history of mental illness, and poor social support. It is better for the Ministry of Health to give training on how to screen depression among medical and surgical patients, and interventions that would address the awareness of the above factors would benefit the prevention of further complications. Further research on risk factors for depression symptoms should be conducted to strengthen and broaden this research.

\section{Abbreviations \\ AMSH: Amanuel Mental Specialized Hospital \\ AOR: Adjusted odds ratio \\ CI: $\quad$ Confidence interval \\ HADS: Hospital anxiety and depression scale \\ OR: Odds ratio \\ PHQ-9: Patient health questionnaire-9 \\ SPHMMC: Saint Paul's Hospital Millennium Medical College \\ SPSS: $\quad$ Statistical Package for the Social Sciences \\ UoG: University of Gondar \\ WHO: World Health Organization.}

\section{Data Availability}

The dataset during and/or analyzed during the current study is available from the corresponding author on reasonable request.

\section{Ethical Approval}

Ethical approval was obtained from the Institutional Review Board of the University of Gondar and Amanuel Mental Specialized Hospital. All participants were given an information sheet and were only included in the study after providing written consent. Confidentiality was maintained by using anonymous questionnaires.

\section{Consent}

Consent is not applicable.

\section{Conflicts of Interest}

The authors declare that they have no competing interests regarding publication.

\section{Authors' Contributions}

Merga S. conceived the study; was involved in the study design, analysis, and report writing; reviewed the article; 
and drafted the manuscript, and GA, HY, and GT were involved in the study design and analysis and drafted the manuscript. All authors read and approved the final manuscript.

\section{Acknowledgments}

The authors acknowledge Saint Amanuel Mental Specialized Hospital, Ethiopia, for funding the study. The authors appreciate the respective study institutions and the study participants for their cooperation in providing the necessary information. The study was funded by the University of Gondar, and the funders are only involved by giving the funding for the design of the study, data collection, analysis, and interpretation.

\section{References}

[1] B. J. Sadock, V. A. Sadock, and P. Ruiz, Kaplane Sadock's Synopsis of Psychiatry Behavioral Sciences/Clinical Psychiatry, Wolters Kluwer, New York, Eleventh edition, 2015.

[2] WHO, "Depression: Let's talk says WHO," As Depression Tops Lists of Causes of Ill Health, Saudi Medical Journal, 2017.

[3] WHO, "Depression and other common mental disorders: global health estimates," Geneva: World Health Organization, 2017, Licence: CC BY-NC-SA 3.0 IGO.

[4] V. Patel, "Talking sensibly about depression," PLoS Medicine, vol. 14, no. 4, 2017.

[5] C. F. Reynolds 3rd and V. Patel, "Screening for depression: the global mental health context," World Psychiatry, vol. 16, no. 3, pp. 316-317, 2017.

[6] M. Friedrich, "Potential cause of nodding syndrome identified," Jamaica, vol. 317, no. 15, p. 1517, 2017.

[7] R. C. Kessler, P. Berglund, O. Demler, R. Jin, K. R. Merikangas, and E. E. Walters, "Lifetime prevalence and age-of-onset distributions of DSM-IV disorders in the National Comorbidity Survey Replication," Archives of General Psychiatry, vol. 62, no. 6, pp. 593-602, 2005.

[8] M. Valstar, B. Schuller, K. Smith et al., "Avec 2014: 3d dimensional affect and depression recognition challenge," Proceedings of the 4th International Workshop on Audio/Visual Emotion Challenge, 2014.

[9] M. Friedrich, "Depression is the leading cause of disability around the world," Jama, vol. 317, no. 15, p. 1517, 2017.

[10] S. Hailemariam, F. Tessema, M. Asefa, H. Tadesse, and G. Tenkolu, "The prevalence of depression and associated factors in Ethiopia: findings from the National Health Survey," International Journal of mental health systems, vol. 6, no. 1, p. 23, 2012.

[11] GBD 2017 Disease and Injury Incidence and Prevalence Collaborators, "Global, regional, and national incidence, prevalence, and years lived with disability for 354 diseases and injuries for 195 countries and territories, 1990-2017: a systematic analysis for the Global Burden of Disease Study 2017," The Lancet, vol. 392, no. 10159, pp. 1789-1858, 2018.

[12] V. Tan, C. Jinks, C. Chew-Graham, E. L. Healey, and C. Mallen, "The triple whammy anxiety depression and osteoarthritis in long-term conditions," BMC family practice, vol. 16, no. 1, p. 163, 2015.

[13] K. Kroenke, J. L. Jackson, and J. Chamberlin PA, MPH, "Depressive and anxiety disorders in patients presenting with physical complaints: clinical predictors and outcome," The American journal of medicine, vol. 103, no. 5, pp. 339-347, 1997.

[14] M. Creamer, D. Wade, S. Fletcher, and D. Forbes, "PTSD among military personnel," International Review of Psychiatry, vol. 23, no. 2, pp. 160-165, 2011.

[15] S. Moussavi, S. Chatterji, E. Verdes, A. Tandon, V. Patel, and B. Ustun, "Depression, chronic diseases, and decrements in health: results from the World Health Surveys," The Lancet, vol. 370 , no. 9590 , pp. 851-858, 2007.

[16] J. Thomas, G. Jones, I. Scarinci, and P. Brantley, “A descriptive and comparative study of the prevalence of depressive and anxiety disorders in low-income adults with type 2 diabetes and other chronic illnesses," Diabetes Care, vol. 26, no. 8, pp. 2311-2317, 2003.

[17] A. J. Mitchell, M. Chan, H. Bhatti et al., "Prevalence of depression, anxiety, and adjustment disorder in oncological, haematological, and palliative-care settings: a meta-analysis of 94 interview- based studies," The Lancet Oncology, vol. 12, no. 2, pp. 160-174, 2011.

[18] P. Austin, S. Wiley, P. M. McEvoy, and L. Archer, "Depression and anxiety in palliative care inpatients compared with those receiving palliative care at home," Palliative \& supportive care, vol. 9, no. 4, pp. 393-400, 2011.

[19] S. Shoar, M. Naderan, M. Aghajani, E. Sahimi-Izadian, N. Hosseini-Araghi, and Z. Khorgami, "Prevalence and determinants of depression and anxiety symptoms in surgical patients," Oman medical journal, vol. 31, no. 3, pp. 176-181, 2016.

[20] N. Khav, T. J. Weiland, G. A. Jelinek, J. C. Knott, and M. Salzberg, "Depression symptoms and risk factors in adult emergency department patients: a multisite cross-sectional prevalence survey," ISRN Emergency Medicine, vol. 2013, 2013.

[21] E. M. Esmayel, M. M. Eldarawy, M. M. Hassan, A. A. Mahmoud, and S. Y. Mohamed, "Mental health problems and sociodemographic correlates in elderly medical inpatients in a university hospital in Egypt," Current Gerontology and Geriatrics Research, vol. 2013, 4 pages, 2013.

[22] M. Denur, G. Tesfaw, and Z. Yohannis, "The magnitude and correlates of common mental disorder among outpatient medical patients in Ethiopia: an institution based cross-sectional study," BMC Research Notes, vol. 12, no. 1, article 360, 2019.

[23] C. K. Maynard, "Differentiate depression from dementia," The Nurse Practitioner, vol. 28, no. 3, pp. 18-19, 2003.

[24] W. J. Katon, "Clinical and health services relationships between major depression, depressive symptoms, and general medical illness," Biological Psychiatry, vol. 54, no. 3, pp. 216226, 2003.

[25] B. Duko, M. Erdado, and J. Ebrahim, "Prevalence and factors associated with depression among hospital admitted patients in South Ethiopia: cross sectional study," BMC research notes, vol. 12, no. 1, p. 73, 2019.

[26] R.-D. Kocalevent, L. Berg, M. E. Beutel et al., "Social support in the general population: standardization of the Oslo social support scale (OSSS-3)," BMC Psychology, vol. 6, no. 1, p. 31, 2018.

[27] G. Tesfaw, G. Ayano, T. Awoke et al., "Prevalence and correlates of depression and anxiety among patients with HIV on-follow up at Alert Hospital, Addis Ababa, Ethiopia," BMC Psychiatry, vol. 16, no. 1, article ???, 2016.

[28] A. Abebe, G. Tesfaw, H. Mulat, and G. Hibdye, "Postpartum depression and associated factors among mothers in Bahir 
Dar Town, Northwest Ethiopia," Annals of General Psychiatry, vol. 18, no. 1, article 19, 2019.

[29] R. Ali, S. Meena, B. Eastwood, I. Richards, and J. Marsden, "Ultra-rapid screening for substance-use disorders: the Alcohol, Smoking and Substance Involvement Screening Test (ASSIST-Lite)," Drug and Alcohol Dependence, vol. 132, no. 1-2, pp. 352-361, 2013.

[30] Group Wb, World Bank Poverity Defintion, 2019.

[31] A. A. Reda, "Reliability and validity of the Ethiopian version of the hospital anxiety and depression scale (HADS) in HIV infected patients," PLoS One, vol. 6, no. 1, p. e16049, 2011.

[32] H. Tilahun, N. Awoke, B. Geda, and F. Mesfin, "Depression and associated factors among adult inpatients at public hospitals of Harari regional state, eastern Ethiopia," Psychiatry Journal, vol. 2018, 6 pages, 2018.

[33] A. Hailu, A. Mandush, K. K. Berhe, H. B. Gebru, and A. Aregay, "Assessment of depression prevalence and its determinants among adult patients admitted in governmental hospitals Mekelle, Tigray, Ethiopia, 2012.; a cross sectional study," International Journal of Pharmaceutical Sciences and Research, vol. 4 , no. 5, p. 1882, 2013.

[34] N. Nakasujja, S. Musisi, J. Walugembe, and D. Wallace, "Psychiatric disorders among the elderly on non-psychiatric wards in an African setting," International psychogeriatrics, vol. 19, no. 4, pp. 691-704, 2007.

[35] A. M. Yohannes, R. C. Baldwin, and M. J. Connolly, "Prevalence of depression and anxiety symptoms in elderly patients admitted in post-acute intermediate care," International Journal of Geriatric Psychiatry, vol. 23, no. 11, pp. 1141-1147, 2008.

[36] A. Morgan, P. Smith, and A. Coore, "The prevalence of depression among medical inpatients at the University Hospitalof the West Indies Jamaica," WIMJ Open, vol. 2, no. 1, p. 33, 2015.

[37] V. Y. de Moraes, M. R. Jorge, F. Faloppa, and J. C. Belloti, "Anxiety and depression in Brazilian orthopaedics inpatients: a cross sectional study with a clinical sample comparison," Journal of Clinical Psychology in Medical Settings, vol. 17, no. 1, pp. 31-37, 2010.

[38] M. A. Cigognini and L. M. Furlanetto, "Diagnosis and pharmacological treatment of depressive disorders in a general hospital," Brazilian Journal of Psychiatry, vol. 28, no. 2, pp. 97103, 2006.

[39] Z. Rukundo, N. Nakasujja, and S. Musisi, "Psychological distress among adults admitted to medical and surgical wards of a Regional Referral Hospital, Uganda," African health sciences, vol. 13, no. 1, pp. 82-86, 2013.

[40] B.-1. Zhong, H.-h. Chen, J.-f. Zhang et al., "Prevalence, correlates and recognition of depression among inpatients of general hospitals in Wuhan, China," General hospital Psychiatry, vol. 32, no. 3, pp. 268-275, 2010.

[41] N. J. Botega, G. N. Mitsuushi, R. C. S. de Azevedo et al., "Depression, alcohol use disorders and nicotine dependence among patients at a general hospital," Revista Brasileira de Psiquiatria, vol. 32, no. 3, pp. 250-256, 2010.

[42] Z. Rukundo, S. Musisi, and N. Nakasujja, "Psychiatric morbidity among physically ill patients in a Ugandan Regional Referral Hospital," African Health Sciences, vol. 13, no. 1, pp. 87-93, 2013. 